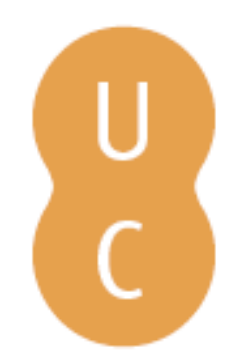

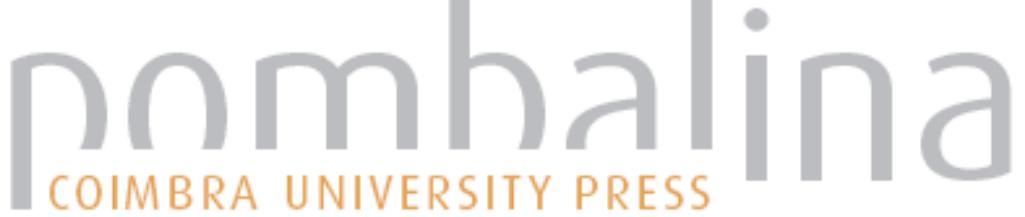

\section{Coordenação comitativa no caboverdiano}
Autor(es):
Brito, José António
Publicado por: Imprensa da Universidade de Coimbra
URL persistente:
URI:http://hdl.handle.net/10316.2/32255
DOI:
DOI:http://dx.doi.org/10.14195/978-989-26-0714-6_6
Accessed : $\quad$ 26-Apr-2023 16:25:43

A navegação consulta e descarregamento dos títulos inseridos nas Bibliotecas Digitais UC Digitalis, UC Pombalina e UC Impactum, pressupõem a aceitação plena e sem reservas dos Termos e Condições de Uso destas Bibliotecas Digitais, disponíveis em https://digitalis.uc.pt/pt-pt/termos.

Conforme exposto nos referidos Termos e Condições de Uso, o descarregamento de títulos de acesso restrito requer uma licença válida de autorização devendo o utilizador aceder ao(s) documento(s) a partir de um endereço de IP da instituição detentora da supramencionada licença.

Ao utilizador é apenas permitido o descarregamento para uso pessoal, pelo que o emprego do(s) título(s) descarregado(s) para outro fim, designadamente comercial, carece de autorização do respetivo autor ou editor da obra.

Na medida em que todas as obras da UC Digitalis se encontram protegidas pelo Código do Direito de Autor e Direitos Conexos e demais legislação aplicável, toda a cópia, parcial ou total, deste documento, nos casos em que é legalmente admitida, deverá conter ou fazer-se acompanhar por este aviso.

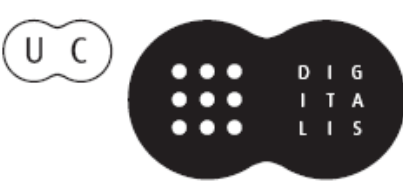




\section{ESTUDOS DE \\ LINGUÍSTICA}

VOLUME II

CONCEIÇÃO CARAPINHA

ISABEL A. SANTOS

COORD.

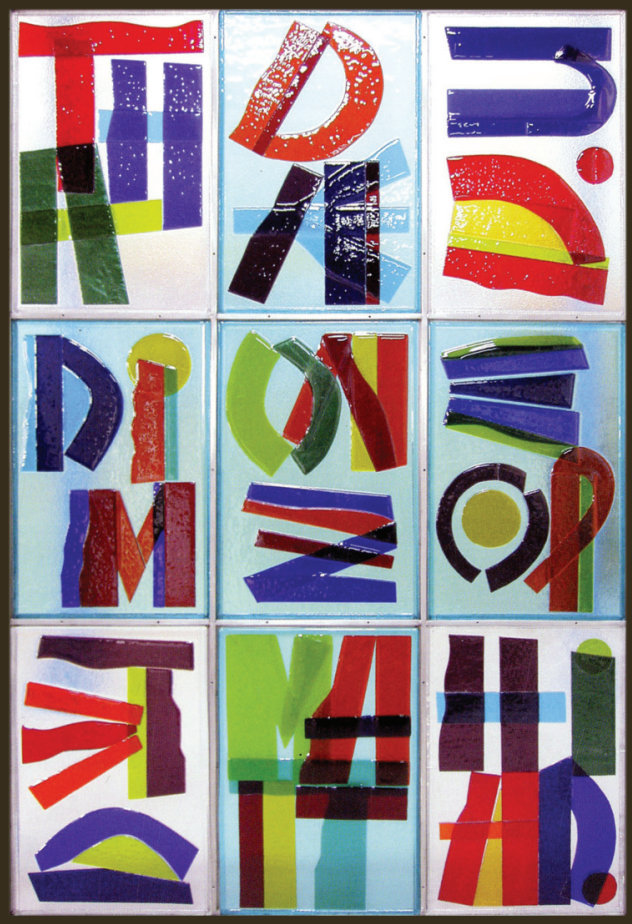




\title{
COORDENAÇÃO COMITATIVA NO CABOVERDIANO 65
}

\author{
José António Brito \\ Faculdade de Letras da Universidade de Lisboa \\ zeantoniobrito@hotmail.com
}

\section{Introdução}

A coordenação comitativa é atestada em diversas línguas do mundo, particularmente nas línguas eslavas, em línguas românicas como o francês, o espanhol e o português ${ }^{66}$.

Nas referidas línguas, caracteriza-se pela possibilidade de um DP (Determiner Phrase) singular se combinar com outro DP por intermédio de um conector tipicamente comitativo para formar um constituinte que, em línguas como o Português Europeu (PE), com marcas de concordância verbal, é um DP composto sintática e semanticamente plural.

Segundo Colaço (2005: 87), "O facto de conduzir à formação de DPs plurais está na base da ideia de que a construção comitativa em causa partilha as propriedades gerais da coordenação, sendo-lhe, em diversos estudos, atribuída a designação de coordenação comitativa”.

65 Este trabalho foi desenvolvido no âmbito da tese de Mestrado realizado na Faculdade de Letras da Universidade de Lisboa, financiado pela Fundação Calouste Gulbenkian (processo $\mathrm{n}^{\circ}$ 950019).

A deslocação para participar no $2^{\circ}$ encontro do CELGA foi financiada pelo grupo ANAGRAMA, do Centro de Linguística da Universidade de Lisboa.

66 Para as línguas eslavas, ver Ladusaw (1989), McNally (1993), Vassilieva / Larson (2005), Skrabalova (2003), etc. Para o Espanhol, ver Camacho (1996, 1999, 2000); para o Português Europeu, ver Colaço (2005). 
Essa partilha de propriedades gerais entre constituintes comitativos e constituintes coordenados varia consoante as línguas, indo de línguas cujo conector coordenativo com valor copulativo utilizado para a coordenação de DPs é o marcador comitativo, como é o caso do Japonês, a línguas como as eslavas (o Russo, o Polaco e o Checo) que, não obstante terem um conector coordenativo copulativo, possuem a chamada coordenação comitativa que, apesar de mais restrita, é muito semelhante à coordenação copulativa, passando por línguas onde a coordenação comitativa é fortemente restringida (Colaço, 2005), como é o caso do Espanhol (Camacho, 1999) e do Português, ou então dependente das propriedades sintáticas e semânticas do verbo, como é o caso do Francês, e também do Português.

Observando os dados do Caboverdiano, constata-se que existem, nesta língua, construções sintáticas com todas as propriedades que usualmente se atribuem à coordenação comitativa, com a particularidade porém, de não se restringir à combinação de DPs, e se estender a constituintes de natureza lexical e sintagmática, com exceção de VP (Verb Phrase) e de constituintes de natureza frásica, como teremos a oportunidade de confirmar no decurso deste trabalho. Além do Caboverdiano, noutras línguas também é possível a conjunção comitativa coordenar categorias distintas de DP. Haspelmath (2004: 19) refere esse facto e ilustra-o com as designadas "WITH-LANGUAGES", como Iraqw, Fongbe, Haitian, Hawsa, Vafsi, Sgaw Karen, Riau, Indonesian, Nêlêmwa e muitas outras línguas da Oceânia ${ }^{67}$.

Neste trabalho, analisaremos os dados do Caboverdiano, com vista a descrever os contextos em que ocorre o conector comitativo com valor conjuncional, bem como o seu comportamento quando comparado com a conjunção coordenativa copulativa.

67 “(...) many of the world's languages use the same marker for expressing conjunctive ('A and $\mathrm{B}^{\prime}$ ) and comitative ('A with $\mathrm{B}^{\prime}$ ) relations (he calls these languages "WITH-languages"). Quite a few of the languages discussed in this volume are WITH-languages in Stassen's sense: Iraqw, Fongbe, Haitian, Hausa, Vafsi, Sgaw Karen, Riau Indonesian, Nêlêmwa and most other Oceanic languages". 


\section{Aspetos que permitem distinguir o uso conjuncional do uso preposicional da partícula comitativa em diversas línguas e em especial em Português Europeu}

Atendendo a que a coordenação comitativa é estabelecida pela presença do conector comitativo que, dependendo do contexto, pode manifestar tanto um comportamento conjuncional como um comportamento preposicional, diversos autores apresentam um conjunto de propriedades que opõem os constituintes que integram uma partícula comitativa com valor conjuncional àqueles em que ocorre uma preposição com valor comitativo. Essas propriedades, além de caracterizarem os comitativos coordenados, são utilizadas como elementos de aproximação entre a coordenação comitativa e a coordenação copulativa canónica. De entre esses aspetos, destacam-se a pluralidade do constituinte resultante, a ligação a anáforas plurais, o controlo das orações não finitas, a obrigatoriedade de adjacência dos termos coordenados, a compatibilidade com o verbo, a obediência à condição da estrutura coordenada ${ }^{68}$, propriedades essas que podem ser conferidas no ponto seguinte para o Caboverdiano.

De momento, uma vez que a partícula comitativa $k u$ teve aparentemente a sua origem na partícula correspondente em Português Europeu com, passamos em revista as propriedades mais relevantes para o Caboverdiano, nomeadamente as do comitativo em PE, designadamente nos aspetos sintáticos e semânticos que a distinguem da construção preposicionada com valor comitativo nessa língua.

Colaço (2005: 89) afirma que, não obstante dispor de uma unidade linguística própria para realizar a coordenação copulativa, o PE permite uma construção em que o comitativo com adquire um valor conjuntivo.

Analisando a distribuição sintática do conector aditivo-comitativo, a autora em apreço afirma que

Em frases com os verbos simétricos, é possível a ocorrência de um PP complemento iniciado pela preposição com, caracterizando-se as frases em que esta construção está presente pelo facto de serem

68 Para mais detalhes sobre este assunto, conferir Colaço (2005). 
semanticamente equivalentes a frases em que os DPs envolvidos ocorrem, em posição de sujeito, unidos pela conjunção copulativa.

Admite ainda que "a mesma possibilidade se verifica relativamente a alguns verbos psicológicos", como por exemplo zangar-se e irritar-se. Subjacente à ideia do uso do conector com valor aditivo ou conjuntivo está, segundo a autora, "a impossibilidade da sua substituição por uma expressão de valor comitativo", aliada à "possibilidade de comutação com a conjunção coordenativa copulativa”, em construções com os verbos referidos.

Segundo a mesma autora, "a ocorrência de um constituinte iniciado por com é também possível em construções com outros verbos, nas quais participa como um constituinte adjunto de natureza preposicional" (Colaço, 2005: 113). Resumidamente, apresenta os seguintes aspetos sintáticos e semânticos que distinguem a coordenação comitativa da construção preposicionada com valor comitativo:

- O conector aditivo-comitativo com só coordena DPs. Ex.: [O ouro com a prata] combinam-se facilmente.

- A coordenação comitativa só ocorre em posição argumental (nunca como adjunto): na posição de sujeito, preferencialmente, mas também na posição de objeto de alguns verbos (que pode ser substituído por um pronome).

Ex.: Fundiram [o ouro com a prata] para fazer um anel.

Fundiram-[nos] para fazer o anel.

- A presença do conector comitativo unindo dois DPs forma uma unidade sintática e semanticamente plural. Ex.: [O Pedro com a Ana] formam um par perfeito.

- A coordenação comitativa requer adjacência dos constituintes articulados.

- A coordenação comitativa só ocorre com predicados de determinada classe (verbos simétricos e psicológicos com valor de reciprocidade entre os argumentos, ser copulativo, verbos leves + predicado que caracterize uma entidade conjunta resultante). 
Concluindo, Colaço assegura que: (i) apenas nos contextos em que ocorre no interior de um complemento verbal o conector comitativo pode manifestar um comportamento conjuncional, exibindo um valor aditivo ou conjuntivo e não puramente comitativo; (ii) quando ocorre no interior de um constituinte adjunto, o conector em questão apresenta um valor comitativo.

\section{Coordenação comitativa no Caboverdiano}

Após passarmos em revista as principais propriedades da coordenação comitativa em diversas línguas, com destaque para aspetos marcantes dessa construção em PE, passamos, de seguida, a abordar esta matéria no Caboverdiano, variante de Santiago.

Na língua Caboverdiana (doravante CV), variante de Santiago, a partícula $k u$ ('com'), dependendo do contexto, afigura-se ora como conjunção coordenativa, ora como preposição.

Colaço (2005: 89) afirma, para o PE, que "apesar de [...] o uso conjuncional da partícula com estar atestado em diversas gramáticas, em nenhuma delas, porém, se propõe a sua inclusão no elenco das conjunções coordenativas." Esta afirmação, todavia, não encontra eco no CV, visto que a lista das designadas "conjunções" apresentada por Veiga (1995: 310-11; 2002: 139) e Baptista (2002) contém este conector.

\subsection{Adjuntos Comitativos e Coordenação Comitativa no Caboverdiano}

Consideremos a seguinte frase:

(1) Djon ku Maria bai Somada y es ben ku pexi. João com Maria ir Assomada e eles vir com peixe 'O João e a Maria foram à Assomada e vieram com peixe.' 
A análise de (1) permite-nos perceber que, em matéria de coordenação, a frase dispõe de mais do que uma unidade linguística para realizar a coordenação aditiva ( $k u$ 'com' e $y$ 'e'). Com efeito, traduzindo-a para o PE, curiosamente, ambos os conectores coordenativos ( $k u$ e $y$ ) são realizados pela conjunção coordenativa copulativa 'e'. Entretanto, não obstante essa coexistência, esses conectores não têm exatamente a mesma distribuição, como veremos mais à frente.

Na frase Djon ku Maria bai Somada y es ben ku pexi, a partícula $k u$ manifesta, em função do contexto, ora comportamento conjuncional, ora comportamento preposicional. Na verdade, em Djon ku Maria, a partícula $k u$ tem um valor aditivo e, por conseguinte, um comportamento conjuncional, equivalente ao da conjunção coordenativa copulativa 'e'. Por seu turno, em es ben $\boldsymbol{k u}$ pexi, a partícula $k u$ é uma preposição, equivalente a 'com'.

Não obstante o uso da mesma unidade linguística $k u$ para exprimir as relações conjuncional e preposicional, no CV, na variante em estudo, existe a conjunção coordenativa copulativa $y$ que, em contextos específicos, como veremos mais adiante, também estabelece a relação de coordenação. Salienta-se o facto de a relação preposicional comitativa ser exclusivamente estabelecida por $k u$. Esta circunstância indicia que provavelmente as duas partículas $k u$, preposicional e conjuncional, terão tido a mesma origem, mas actualmente apresentam propriedades que as distanciam. Com efeito, em Djon ku Maria, ku veicula a ideia de adição, e o resultado é equivalente a situações em que os DPs envolvidos ocorrem em posição de sujeito, agregados pela conjunção coordenativa copulativa, denotando a inclusão conjunta de duas entidades numa entidade, enquanto em es ben ku pexi, ocorre uma preposição comitativa $k u$ num constituinte adjunto. A natureza preposicional desse conector pode ser atestada pela possibilidade de substituição de $k u$ por sen, ilustrada na frase seguinte:

(2) Es ben sen pexi.

Eles vir sem peixe

'Eles vieram sem peixe.' 


\subsection{Obediência da coordenação comitativa no Caboverdiano às propriedades caracterizadoras da coordenação comitativa}

A coordenação comitativa caracteriza-se por um conjunto de propriedades: a pluralidade do constituinte resultante, a ligação de anáforas plurais, o controlo das orações não finitas, a obrigatoriedade de adjacência dos termos coordenados, a compatibilidade com o verbo, a obediência à condição da estrutura coordenada. Nesta secção, veremos como elas se manifestam no $\mathrm{CV}$, considerando os casos prototipicamente referidos na literatura.

\section{(a) Pluralidade do constituinte resultante}

Colaço (2005: 96) afirma que, numa estrutura de coordenação em que ocorre a partícula comitativa com valor conjuncional, o constituinte nominal coordenado resultante é plural, à semelhança do que acontece com a estrutura de coordenação copulativa.

A pluralidade da forma verbal, que decorre da presença de sujeito com uma estrutura coordenada, é utilizada para diferenciar o uso conjuncional do uso preposicional do conector comitativo com, uma vez que este último não conduz à pluralidade da forma verbal.

No CV não há marcas de concordância sujeito-verbo, uma vez que os verbos não têm flexão de pessoa ou número e só admitem, restritamente, marcas de TMA (tempo, modo e aspeto). No entanto, se se recorrer a testes de constituência, como a substituição por pronome pessoal ou a retoma anafórica, conclui-se que, efetivamente, a ocorrência de uma partícula comitativa com valor conjuncional conduz à pluralidade do constituinte que a integra, como confirmam os exemplos (3), (4) e (5):

(3)a. [...] bu pai ku bu mai resebe-u [...] teu pai com tua mãe receber tu 'O teu pai e a tua mãe receberam-te.' 
(3) $\mathrm{a}^{\prime}$. [...][bu pai ku bu mai] resebe-u [...]

Teu pai com tua mãe receber tu

'O teu pai e a tua mãe receberam-te.'

(4)a. [...] [Es] resebe-u $[\ldots]$

Eles receber tu

'Eles receberam-te.'

(4) $\mathrm{a}^{\prime} .[\ldots] * \mathrm{E}$ recebe-u.

Ele receber tu

*'Ele receberam-te.'

Substituindo [bu pai ku bu mai] por um pronome pessoal - cf. (4) -, o único resultado aceite é es ('eles'), ou seja, a terceira pessoa do plural, como mostram os seguintes exemplos:

O contraste entre os exemplos ( 4 a.) e ( $4 \mathrm{a}^{\prime}$.) confirma que o sujeito [bu pai ku bu mai] é um DP plural resultante de uma estrutura de coordenação estabelecida pela partícula comitativa $k u$ com valor conjuncional.

$\mathrm{Na}$ linha do teste de substituição (por um pronome), o teste de retoma anafórica em estruturas de coordenação também confirma que apenas es ('eles'), terceira pessoa do plural, pode ser retomado como correferente do sujeito [bu pai ku bu mai] (cf. (5 a.) e (5 b.)).

(5)a. [...] [bu pai ku bu mai $]_{\mathrm{i}}$ resebe-u, mas $[\text { es }]_{\mathrm{i}}$ txora. Teu pai com tua mãe receber tu mas eles chorar 'O teu pai e a tua mãe receberam-te, mas choraram.'

(5)b *[...] [bu pai ku bu mai $]_{\mathrm{i}}$ recebe-u, mas $[e]_{\mathrm{i}}$ txora. Teu pai com tua mãe receber tu mas ele/ela chorar *'O teu pai e a tua mãe receberam-te, mas ele/ela chorou.' 
O teste de constituência permite averiguar que o constituinte [ $b u$ pai ku bu mai] veicula, efetivamente, a noção de pluralidade, verificável a partir do recurso à terceira pessoa do plural es ('eles') para o substituir (cf. (5 a.)), e da agramaticalidade da sua substituição por $\boldsymbol{e}$ ('ele'), terceira pessoa do singular (cf. $(5$ b.)).

Por razões já referidas, o DP [bu pai ku bu mai], apesar de semântica e sintaticamente plural, não desencadeia a concordância verbal, não obstante estar na posição do sujeito.

\section{(b) Ligação a anáforas plurais}

Os exemplos (6) permitem confirmar que, no CV, a presença de uma partícula comitativa com valor conjuncional numa estrutura coordenada possibilita, ao DP que a integra, o controlo de anáforas plurais.

(6)a. [Ana ku Maria $]_{\mathrm{i}}$ odja $\left[\begin{array}{ll}\text { ses } & \text { kabesa }\end{array}\right]_{\mathrm{i}}$ na spedju. Ana com Maria olhar suas cabeça na espelho 'A Ana e a Maria viram-se no espelho.'

(6)b. * [Ana ku Maria $]$ odja $[\text { si kabesa }]_{\mathrm{i}}$ na spedju. Ana com Maria olhar sua cabeça na espelho *'A Ana e a Maria viu-se no espelho.'

Em (6)a., a anáfora ses kabesa (correspondente ao se reflexo no PE) toma obrigatoriamente o DP Ana ku Maria como antecedente, ou seja, obedece ao Princípio A da Teoria da Ligação. Em (6)b., no entanto, o reflexo si kabesa (no singular) não pode ter como antecedente o DP Ana ku Maria visto que não veicula a ideia de pluralidade contida nesse DP. 


\section{(c) Teste Controlo de sujeito de orações encaixadas não finitas}

O teste de controlo de sujeito de orações encaixadas não finitas prova que a partícula comitativa tem um comportamento conjuncional e não de preposição com valor comitativo e que a conjunção comitativa tem um valor aditivo, como elucida o exemplo seguinte:

(7) Djon ku Maria kre/tenta/prumete PRO ruspeta kunpanheru João com Maria querer/tentar / prometer respeitar companheiro 'O João e a Maria querem /tentam / prometem respeitar-se.'

Em (7), só temos kunpanheru (correspondente ao 'se' recíproco em português) na posição de objeto da oração encaixada se o sujeito da oração matriz, que controla o sujeito da encaixada não finita, for complexo. Isto prova que a partícula comitativa tem um comportamento conjuncional e não de preposição com valor comitativo.

\section{(d) Obrigatoriedade de adjacência dos termos coordenados}

Colaço (2005) afirma que o constituinte antecedido da partícula comitativa com valor conjuncional ocorre obrigatoriamente em adjacência ao DP com o qual está relacionado. No Caboverdiano verifica-se esta propriedade, como provam os exemplos ( 8 a.) e ( 8 b.):

(8)a. [DP [DP Ana] ku [DP Maria]] kunpra pexi.

Ana com Maria comprar peixe

'A Ana e a Maria compraram peixe'.

b. *[Ana] kunpra pexi [ku Maria]

Ana comprar peixe com Maria *'A Ana comprou peixe com Maria'

O conector $k u$ tem estatuto de um clítico que 'proclisa' o termo coordenado que o segue. Haspelmath (2004: 6) afirma que em alguns 
idiomas o conector coordenativo é fonologicamente dependente de um dos termos coordenados ${ }^{69}$.

A não adjacência ao DP com o qual está relacionado implica uma leitura não grupal e, no exemplo em análise, conduz a uma frase mal formada.

O contraste ente $(8 \mathrm{a}$.) e ( 8 b.) confirma a propriedade supramencionada. Com efeito, a violação da obrigatoriedade da adjacência resultou na agramaticalidade de ( 8 b.).

\section{(e) Compatibilidade com o verbo}

Em diversas línguas, a estrutura de coordenação é descrita como independente das propriedades do verbo, diferentemente do que acontece em PE. No CV, como evidenciam os exemplos (9), a estrutura de coordenação que envolve a partícula comitativa não depende das propriedades do verbo:

(9)a [Ana ku Lita $]_{\text {EXPERIENCIADOR }}$ sabe $\left.\left.[\text { kriolu }]_{\text {TEMA }}\right]\right]$. Ana com Lita saber crioulo

'A Ana e a Lita sabem crioulo.'

b. [Ana ku Lita staba duenti

Ana com Lita estar doente

'A Ana e a Lita estavam doentes.'

Como se deduz da análise dos exemplos apresentados, nem (9 a.) nem (9 b.) apresentam verbos que pressupõem agentividade ${ }^{70}$. Em (9 a.), sabe ('saber') é um verbo epistémico de dois lugares, com um Experienciador e um Tema; em (9 b.), temos um verbo copulativo, razão por que enquadramos o CV no grupo de línguas em que a estrutura de coordenação

69 Texto original: "In some languages, the coordinator is clearly phonologically attached to one of the coordinands, either as a proclitic or as an enclitic (...)".

$70 \mathrm{O}$ constituinte comitativo adjunto não pode ocorrer com verbos que não pressupõem agentividade. 
envolvendo a partícula comitativa não depende das propriedades semânticas dos verbos.

\section{(f) Obediência à Condição da Estrutura Coordenada}

Ross (1967: 89) propôs, na sua tese, a restrição designada Coordinate Structure Constraint [Condição de Estrutura Coordenada (CEC)], que proíbe o movimento de qualquer constituinte para fora do sintagma coordenado: "In coordinate structure, no conjunct may be moved, nor may any element contained in a conjunct be moved out of that conjunct."

No CV, como elucidam os exemplos (10 a.) e (10 b.), o desrespeito por essa condição produz agramaticalidade:

(10)a.[Ana ku Maria] odja ses kabesa na spedju. Ana com Maria olhar suas cabeça na espelho 'A Ana e a Maria viram-se no espelho.'

6. $\quad{ }^{*}$ Ku Maria, Ana odja ses kabesa na spedju.

Com Maria Ana olhar suas cabeças na espelho *'Com a Maria, a Ana viram-se no espelho.'

O exemplo (10 a.) obedece à referida CEC e o resultado é uma frase bem formada. Em (10 b.), o segundo termo coordenado foi deslocado para o início da frase, violando a CEC, originando uma frase agramatical.

Em síntese, a submissão da partícula comitativa $k u$ ao teste de verificação das propriedades caracterizadoras da coordenação comitativa permitiu-nos confirmar que o $k u$ CV é efetivamente uma conjunção comitativa, visto que responde positivamente a esse teste, confirmando a sua aproximação à conjunção coordenativa copulativa $y$ ' $e$ '. 


\section{Coordenação comitativa versus coordenação copulativa canónica no PE e em CV}

A coordenação comitativa partilha as propriedades gerais da coordenação. Apesar das semelhanças entre as estruturas resultantes do uso conjuntivo do conector comitativo e as estruturas de coordenação copulativa canónica, existem algumas propriedades que permitem diferenciá-las em diversas línguas, como o português. Essas propriedades são ${ }^{71}$ :

\section{(a) Reiteração da partícula comitativa}

Ao contrário do que acontece com a coordenação copulativa canónica em diversas línguas, a coordenação comitativa não é iterativa no português. Não obstante esta constatação, há registo de que, nas chamadas with-languages ${ }^{72}$ , a conjunção comitativa pode ser reiterada. Neste particular, o CV, ao contrário do que acontece com o PE, integra o grupo de línguas em que a conjunção comitativa é iterativa. O exemplo (11) ilustra essa característica da coordenação comitativa na língua em análise.

\section{(11)a.Palu ku Pedru Ku Manel ku Litu bai Praia. \\ Paulo com Pedro com Manuel com Lito ir Praia \\ 'O Paulo, o Pedro, o Manuel e o Lito foram à Praia.'}

Em alternativa a este tipo de construção, usa-se a coordenação assindética, em vez da coordenação copulativa $y$. Veja-se o contraste da gramaticalidade das frases seguintes:

(12)a.Palu, Pedru, Manel, Litu bai Praia.

Paulo, Pedro, Manuel, Lito ir Praia

'O Paulo, o Pedro, o Manuel, o Lito foram à Praia.'

\footnotetext{
${ }^{71}$ Para detalhes sobre estas propriedades, conferir Colaço (2005).

72 Stassen (2001) divide as línguas do mundo em dois grupos: and-languages e withlanguages. Para esse autor, as with-languages dispõem apenas de estratégias comitativas para realizar a coordenação copulativa.
} 
b. *Palu y Pedru y Manel y Litu bai Praia.

Paulo e Pedro e Manuel e Lito ir Praia

O Paulo e o Pedro e o Manuel e o Lito foram à Praia.'

Estes exemplos reforçam a diferença comportamental entre as coordenações comitativa e copulativa em CV e em PE.

\section{(b) Restrições categoriais aos termos coordenados}

Colaço (2005: 101) afirma: "Parece ser comum à quase totalidade das línguas atestadas que dispõem de coordenação comitativa o facto de estar limitada categorialmente a DPs, podendo ser unicamente esta a categoria de qualquer dos termos coordenados."

Entretanto, como atestam os exemplos em baixo, no CV, além de DPs, a coordenação comitativa aplica-se também a AdvPs (adverbial phrase), APs (Adjectival Phrase), PPs (Prepositional Phrase) e frases não finitas. Ao não impor muitas restrições à categoria dos constituintes que seleciona como termos de coordenação, a partícula comitativa $k u$ ('com') manifesta "o comportamento transcategorial, característico da maioria das conjunções coordenativas" 73 .

- Coordenação de DPs e de Pronomes:

(13)a.[DP[NPAna] $\boldsymbol{k u}$ [NP Maria] sta xintadu.

Ana com Maria estar sentado

'A Ana e a Maria estão sentadas.'

b. [Mi] ku[ bo] nu ta studa djuntu.

Eu com tu nós TMA estudar junto

'Eu e tu estudamos juntos.'

73 Para Colaço (2004: 448), assim se explica o comportamento transcategorial da maioria das conjunções coordenativas: não impondo restrições às categorias dos constituintes que seleciona como termos da coordenação, o núcleo Conj assume a sua natureza categorial, que se projeta, naturalmente, até ConjP. 
- Coordenação de AdvPs

(14) [AdvP Onti] $\boldsymbol{k u}$ [AdvP oji] faze friu.

Ontem com hoje fazer frio

'Ontem e hoje fez frio.'

- Coordenação de APs

(15) Maria tene [NP [buluza risku-risku [AP azul] $\mathbf{k u}$ [AP branku]]. Maria tem blusa risco-risco azul com branco

'A Maria tem uma blusa azul e branca às riscas.'

- Coordenação de PPs

(16) Maria faze dosi [pp di koku] ku [pp di papaia].

Maria fazer doce de coco com de papaia.

'A Maria fez doces de coco e de papaia.'

- Coordenação de Verbos não finitos com valor nominal

(17) [Le] ku [skrebe] e sinplis.

Ler com escrever é simples

'Ler e escrever é simples.'

Contudo, a coordenação comitativa não é possível nos seguintes casos: VPs e Frases (finitas). Os exemplos abaixo ilustram esta afirmação.

- Coordenação de VPs

(18) *Djon ta $\hat{\mathrm{e}} \mathrm{ku}$ ta skrebe kriolu. João TMA ler com TMA escrever crioulo 'O João lê e escreve em crioulo.' 
- Coordenação de Frases (finitas)

(19) *Ana le livru ku skreve puema.

Ana ler livro com escrever poema

'A Ana leu o livro e escreveu o poema.'

Salienta-se que em contextos de coordenação de VPs, APs em posição predicativa e Frases, a alternativa é a coordenação assindética ou a utilização da conjunção coordenativa copulativa $y .{ }^{74}$

\section{Conclusão}

Os exemplos acima apresentados comprovam que a coordenação comitativa é bastante produtiva no Caboverdiano e que abarca diversas categorias como AdvP, PPs, DPs, etc.

Os dados analisados permitem-nos verificar que o Caboverdiano dispõe, à semelhança do que acontece com muitas línguas, de coordenação comitativa, realizada através do conector comitativo $k u$ ('com'). Nesta língua, a coordenação comitativa não se restringe à combinação de DPs, pois abarca diversas categorias como DPs, PPs, AdvPs, e Verbos não-finitos com valor nominal.

Não obstante o conector comitativo $k u$ corresponder etimologicamente a com (português), sintaticamente estes dois elementos apresentam diferenças substanciais. Se os aspetos que permitem distinguir o uso conjuncional do uso preposicional da partícula comitativa têm muito em comum, já as relações que essas unidades estabelecem com a coordenação copulativa canónica diferem substancialmente, nomeadamente quanto à reiteração da partícula comitativa e às restrições categoriais aos termos coordenados. Enquanto no PE a coordenação comitativa é bastante restritiva e, de algum modo, marginal, no Caboverdiano, a coordenação comitativa é

${ }^{74}$ Este aspeto está fora do âmbito deste trabalho, por razões que se prendem com limitações de espaço. 
bastante produtiva, sobretudo em projeções que não envolvem núcleos verbais, instâncias em que prevalece o uso da coordenação assindética, ou núcleos predicativos e frases, onde ocorre a coordenação assindética ou a conjunção coordenativa copulativa $y$.

\section{Referências bibliográficas}

Baptista, Marlyse (2002): The Syntax of Cape Verdean Creole: The Sotavento Varieties. In: Linguistics Today, 54.

Camacho, J. (1996): Comitative Coordination. In: Parodie, Claudia t alli (org.): Aspects of Romance Linguistics: Selected Papers from the Linguistics Symposium on Romance Linguistics XXIV. Washington, D. C.: Georgetown University Press, 107122.

Camacho, J. (1999): La Coordinación. In: Bosque / Demonte (orgs.): Gramática Descriptiva de la Lengua Española, vol. 2. Madrid: Editorial Espasa Calpe, 2635-2694.

Camacho, J. (2000): Structural Restrictions on Comitative Coordination. In. Linguistic Inquiry, $31,2,366375$.

Colaço, Madalena (2004): Coordenação comitativa em Português Europeu. In: Actas do XIX Encontro da Associação Portuguesa de Linguística, 383-396.

Colaço, Madalena (2005): Configurações de coordenação aditiva: tipologia, concordância e extracção. Dissertação de Doutoramento em Linguística apresentada à Universidade de Lisboa.

Haspelmath, Martin (ed.), (2004): Coordinating constructions. Amsterdam: John Benjamins, 339.

Ladusaw, W. (1989): Group Reference and the Plural Pronoun Construction. In: Papers on the Plural Pronoun Construction and Comitative Coordination, UCSC Syntax Research Center Report SRC-89-02). UCSC Syntax Research Center, Santa Cruz, CA, 1-7.

McNally, L. (1993): Comitative Coordination: a Case Study in Group Formation. In: Natural Language and Linguistic Theory, 11:2, 347379.

Ross, J. R. (1967): Constraints on variables in syntax. Doctoral dissertation, MIT, Cambridge, Massachusetts.

Skrabalova, H., 2003: Comitative constructions in Czech. In Kosta, P. et al. (edd.): Formal Investigations Into Slavic Languages. Francfort sur le Main: Peter Lang. 685-696.

Stassen, L. (2001): AND-languages and WITH-languages. In: Linguistic Typology, 4.1., 155.

Vassilieva, M. \& R. K. Larson (2005): The Semantics of the Plural Pronoun Construction. In: Natural Language Semantics, 13, 101-124.

Veiga, Manuel (1995): O Crioulo de Cabo Verde: introdução à gramática. Praia: Instituto Caboverdiano do Livro.

Veiga, Manuel (2002): O Caboverdiano em 45 Lições. Praia: INIC.

Veiga, Manuel (2009): Odju d’Agu. Praia: Instituto da Biblioteca Nacional e do Livro. 